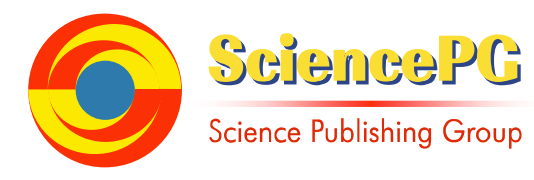

\title{
An Entropy-Based MIMO Array Optimization for Short-Range UWB Imaging
}

\author{
Li Zhi ${ }^{1}$, Zhang Jianwen ${ }^{2}$, Shen Yu ${ }^{1}$, Hu Jun ${ }^{1}$ \\ ${ }^{1}$ Department of Communication and Command, Chongqing Communication College, Chongqing, China \\ ${ }^{2}$ Department of Clinical Medicine, Chongqing Medical and Pharmaceutical College, Chongqing, China
}

\section{Email address:}

lizhi_cs@126.com (Li Zhi), macsea@126.com (Zhang Jianwen)

\section{To cite this article:}

Li Zhi, Zhang Jianwen, Shen Yu, Hu Jun. An Entropy-Based MIMO Array Optimization for Short-Range UWB Imaging. International Journal of Wireless Communications and Mobile Computing. Vol. 4, No. 2, 2016, pp. 32-36. doi: 10.11648/j.wcmc.20160402.14

Received: April 6, 2016; Accepted: April 29, 2016; Published: May 9, 2016

\begin{abstract}
A novel approach to design the position of linear Multiple-Input Multiple-Output (MIMO) array elements for short-range UWB imaging is proposed. The proposed method uses Particle Swarm Optimization (PSO) algorithm to determine the optimal MIMO antenna array topologies that can provide minimum entropy of the reconstructed image. According to the approach, a MIMO array is optimized with minimum entropy indicating high focusing quality.
\end{abstract}

Keywords: Array Design, UWB, MIMO, Entropy, PSO

\section{Introduction}

As an emerging technology, short-range UWB imaging has the ability to penetrate through obstacles with high precision and low electromagnetic radiation. It has many applications, such as through wall imaging (TWI) [1], security inspection [2] and ground penetrating radar (GPR) [3]. By taking the advantages of MIMO technique [4, 5], short-range UWB imaging can reduce lots of antennas, which releases the total cost and weight of the sensor system.

In short-range UWB imaging application, array design is one of the key techniques, and it can influence target detection, parameters estimation and imaging performance such as the resolution and sidelobe level of the forming image. Usually, for imaging cases, resolution in the range direction can be achieved by transmitting wide band signals, and resolution in the cross-range direction can only be achieved by varying the illumination over the field of view of the sensor system. But for short-range application, the traditional pattern design method that based on plane wave is limited for the space-variance effect, so the imaging is determined by both wide band signals and short-range effect, which means that the resolution in range direction and cross-range direction is crossed. Thus, how to design a proper array transmitting specified signals (e.g. the frequency band for TWI is from $250 \mathrm{MHz}$ to $3 \mathrm{GHz}$ [6]) is on focus. In some experiments $[7,8]$, a uniform linear array is used to form the image. It is simple but not the best configuration. In some other papers, a random distributed array is discussed that it could reduce the effect of ghost images [9, 10]. But not all random patterns produce good results [9].

As the short-range effect and wide band property, it is difficult to design an optimized MIMO array. But on the other hand, the antenna array is used for high quality images, so it is direct and feasible to optimize array by the imaging performance. In this paper, the optimization of a linear MIMO array for short-range UWB imaging application is investigated. To find the best positions of antenna elements within the array, which provide desired resolution and sidelobe level, the PSO algorithm is used. By using PSO with proper optimization objective, which is the entropy of image that indicates the focusing quality and constraints on the fixed number of antenna elements and the probable maximal baseline, we can determine the proper antenna element positions.

This paper is organized as follows. The antenna array, imaging method and Point Spread Function (PSF), which is defined as the image distribution of a point scatter over the area of interest, are described in section 2 . In section 3 , the proposed approach is illustrated. In section 4, the optimized MIMO array and its corresponding results are shown. Finally, conclusions can be found in section 5 . 


\section{Imaging}

\subsection{Antenna Array}

In a conventional narrow-band radar system with a linear array, the cross-range resolution is solely determined by the size of the array aperture, and the sidelobe level is associated with the space between elements. When the number of elements is given, the conflict between the aperture size and the elements space corresponds to the conflict of the mainlobe width and the sidelobe level, which should be a trade-off. However, in UWB radar systems, the cross-range resolution is related not only to the baseline size, but also the bandwidth and the pulse shape of the system [11, 12, 13]. Hence, the cross-range resolution is a function of not only the array geometry, but also the spectrum of the radiated signal. Furthermore, the sidelobe level at range direction and cross range direction is also influenced by the pulse shape. As a result, the desired resolution may be achieved by modifying the transmitted pulse spectrum to achieve a certain PSF, as in [14], or by modifying the antenna topology, or both. In this paper, we only concentrate on the influence of antenna array topology to the PSF, so we fix the bandwidth and the pulse shape, and optimize the resolution by adjusting the antenna elements positions.

To obtain the image with real aperture requires large number of elements and multiple channels in transceiver. To avoid too much cost, electronics fabrication problems, and large data flow for real-time imaging, the antenna array must be thinned. However, aperture thinning generally causes the raise of sidelobe level. Thinned arrays have been designed and used as a means of obtaining maximum resolution with a minimal number of elements, using the coarray as a means of quantifying performance [11]. A good alternative array thinning can be MIMO approach. It was demonstrated in [12] and [15] that for a MIMO antenna array, the equivalent aperture is the spatial convolution of the transmitting antenna array aperture and the receiving antenna array aperture. What's more, each frequency responds to one equivalent aperture, which forms a disperse coarray $[15,16]$. As a result, the antenna aperture size and element spacing of the effective array can be much larger and denser than those of the monostatic antenna array, providing better mainlobe and sidelobe control. The advantages of using MIMO antenna arrays are also demonstrated in [5]. So in our program, a MIMO array is applied.

\subsection{Imaging Algorithm}

In this paper, the image is formed by Back Projection (BP) algorithm, which can be calculated with high resolution in time domain and makes no geometrical approximations [17, 18]. BP algorithm is very suitable for short-range UWB imaging, such as TWI and GPR [4, 17].

If $t$ denotes fast time, $\mathbf{x}_{t m}$ denotes the location of transmitter $m, m=1, \ldots, M, \mathbf{x}_{r n}$ denotes the location of receiver $n, n=1, \ldots, N$, and $s_{m n}(t)$ represents the signal transmitted by transmitter $m$ and received by receiver $n$, then according to the BP algorithm the amplitude at the image pixel $\mathbf{x}_{q}$ is given by

$$
\mathrm{I}\left(\mathbf{x}_{q}\right)=\sum_{m=1}^{M} \sum_{n=1}^{N} w_{r n} \cdot s_{m n}\left(\frac{\left\|\mathbf{x}_{t m}-\mathbf{x}_{q}\right\|+\left\|\mathbf{x}_{q}-\mathbf{x}_{r n}\right\|}{v}\right)
$$

Where $w_{r n}$ is the weight of receiver $n, v$ is the velocity of the electromagnetic wave in the ambient medium. The coherent sum is performed via time-shifting the signal obtained by each antenna pair and then adding across all antenna pairs to integrate the value at the image space pixel $\mathbf{x}_{q}$. This time shift and sum sequence is repeated for all the image space pixels. For an ideal point target, the calculated image is its PSF [19].

\section{Methodology}

Particle Swarm Optimization (PSO) is a biologically inspired computational search and optimization method developed in 1995 by Eberhart and Kennedy based on the social behaviors of birds flocking or fish schooling [20]. PSO has been used by many applications of several problems and has a great success. PSO is a sort of evolutionary algorithm utilizing swarm intelligence to achieve the goal of optimizing a specific fitness function, and it operates on a model of social interaction between independent particles [20]. PSO consists of a swarm of particles, where one particle represents a potential solution. Exploration is the ability of a search algorithm to explore different region of the search space in order to locate a good optimum. Exploitation, on the other hand, is the ability to concentrate the search around a promising area in order to refine a candidate solution [21]. With their exploration and exploitation, the particles of the swarm fly through hyperspace and have two essential reasoning capabilities: memory of their own best position -- local best, and knowledge of their neighborhood's best -- global best. With a velocity, each particle could change its position to explore in the solution space.

According to the PSO algorithm, the position $x_{k}(i)$ and velocity $v_{k}(i)$ of the particles are updated at each iteration $i$ according to the velocity and position update equations given by

$$
\begin{aligned}
v_{k}(i+1)= & v_{k}(i)+c_{1} \cdot \xi_{1} \cdot\left(\text { localbest }_{k}(i)-x_{k}(i)\right) \\
& +c_{2} \cdot \xi_{2} \cdot\left(\text { globalbest }(i)-x_{k}(i)\right) \\
& x_{k}(i+1)=x_{k}(i)+v_{k}(i)
\end{aligned}
$$

Where $k=1,2, \ldots, K, k$ is the total particle number in the swarm, $x_{k}(i)$ and velocity $v_{k}(i)$ are the position and velocity vectors, and localbest $_{k}(i)$ and globalbest $(i)$ represent the local and global best position in the swarm. $\xi_{1}, \xi_{2}$ is the random 
variable between 0 and 1 , and $c_{1}, c_{2}$ is the optimization parameter which is dependent on the particle's movement and usually set to be 2 , that is $c_{1}=c_{2}=2$. In each dimension, the particle has a limited maximum velocity $V_{\max }$, and if the velocity exceeds $V_{\max }$, the velocity is set to be $V_{\max }$.

The entropy of a radar image is an indicator of its focusing quality. As the image is blurred, the uncertainty in the location and dimension of a target increases. In this paper, the Shannon entropy was calculated to measure the focusing quality. The best focusing quality is achieved when the entropy of the reconstructed image is minimized [22, 23], and the corresponding position of the array element is regarded as optimal.

The focusing quality of the image depends on the array element positions during the reconstruction process, and it can be used as a metric. This metric measures the level of uncertainty in a random variable. Let $r$ be a discrete random variable with a probability density function $p(r)$. According to Shannon's definition [24], the entropy of $r$ is given by

$$
H=-\sum p(r) \log (p(r))
$$

The entropy of a digital image with $W$ intensity levels is defined as

$$
H=-\sum_{w=1}^{W} \frac{\psi_{w}}{\psi} \log \left(\frac{\psi_{w}}{\psi}\right)
$$

Where $\psi_{w}$ are the pixel number corresponding to the $w$ th intensity level on the image and $\psi$ is the total number of pixels in the image. The image that has the minimum entropy has the optimal focusing quality. The entropy of the image is used as the fitness function of PSO algorithm to find the corresponding array element positions with some specific constraints.

The proposed design method can be described as follows.

a) Set the parameters of the imaging scenery and array constraints, such as the element number of the MIMO array and the probable maximum length of the array.

b) Generate the initial population with random array element position $x_{1}, x_{2}, \ldots x_{k}, \ldots x_{K}$, where $K$ is the size of the particle swarm. The particle $k$ and the velocity at iteration $i$ are denoted as $x_{k}(i)$ and $v_{k}(i)$. Here $i=0$ at the beginning. Initially all of the particle velocity is assumed to be zero. Thus the particles has the initial status $x_{k}(0)$ and $v_{k}(0)$.

c) Evaluate the fitness function value for each particle as $f\left[x_{1}(i)\right], f\left[x_{2}(i)\right], \ldots f\left[x_{k}(i)\right], \ldots f\left[x_{N}(i)\right]$. The fitness function is based on the entropy of the image and $f\left[x_{k}(i)\right]=-H, H$ is calculated by equation (5).

d) For each particle $k$, compare its fitness function value $f\left[x_{k}(i)\right]$ with $P_{b e s t}(k)$, which is the best fitness function value of particle $k$ at all previous iteration (Initialize as $\left.P_{\text {best }}(k)=-\infty\right)$ ). If $f\left[x_{k}(i)\right]>P_{b e s t}(k)$, that means $f\left[x_{k}(i)\right]$ is better than $P_{\text {best }}(k)$, then let $P_{b e s t}(k)=f\left[x_{k}(i)\right]$ and localbest $_{k}(i)=x_{k}(i)$.

e) For each particle $k$, compare its fitness value $f\left[x_{k}(i)\right]$ with $G_{b e s t}$, which is the best fitness function value of all particles at all previous iteration(Initialize as $\left.G_{\text {best }}=-\infty\right)$. If $f\left[x_{k}(i)\right]>G_{\text {best }}$, that means $f\left[x_{k}(i)\right]$ is better than $G_{b e s t}$, then let $G_{b e s t}=f\left[x_{k}(i)\right]$ and globalbest $_{k}(i)=x_{k}(i)$.

f) Update $x_{k}(i), k=1,2, \ldots, K \quad$ and $\quad v_{k}(i), k=1,2, \ldots, K$ according to equation (2) and (3).

g) Check whether the current solution is convergent. This iteration process continues until all particles convergence the same solution or the iteration $i$ reaches a specific number. The convergence is determined by the termination criteria that the difference between the current solution and the previous one is very small. If not convergent then step (c) is repeated by updating iteration $i=i+1$.

\section{Results}

In the following short-range UWB imaging simulation, the configuration of a linear MIMO array is optimized by PSO algorithm with the corresponding image entropy as its cost function. The MIMO array has 3 transmitting elements and 10 receiving elements. The aperture of the linear MIMO array is limited to shorter than 2 meters. The transmitting signal is orthogonal stepped frequency continuous wave (SFCW) [25] with a center frequency of $1.5 \mathrm{GHz}$ and a bandwidth of $2 \mathrm{GHz}$, and the signal duration is $1 \mu \mathrm{s}$. The imaging area is 8 meters along azimuth and 4 meters along range. An ideal point target at the center of the imaging area is used as the scatter, and the PSF formed by BP algorithm is used to calculate the entropy.

The optimization is executed on MATLAB platform. Fig. 1 shows the optimized MIMO array, in which red * represents transmitting antenna element, blue dot represents receiving antenna element. The topology indicates that the MIMO array has a transmitting array aperture expanding to the largest length as well as the receiving array aperture. This result is consistent with the coarray theory that the outer elements provide better resolution [13, 15]. Fig. 2 shows the corresponding PSF. Rather than a uniform array, the distributed elements provide sidelobe with low level. The entropy changes following the iteration as shown in Fig. 3. From the figure it can be seen that the entropy declines to lower and lower level, then close to steady. The focusing quality gets better and better following with the iteration.

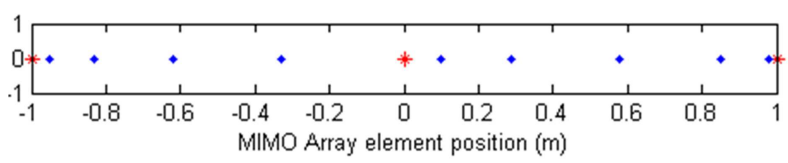

Fig. 1. the optimized MIMO array topology. 


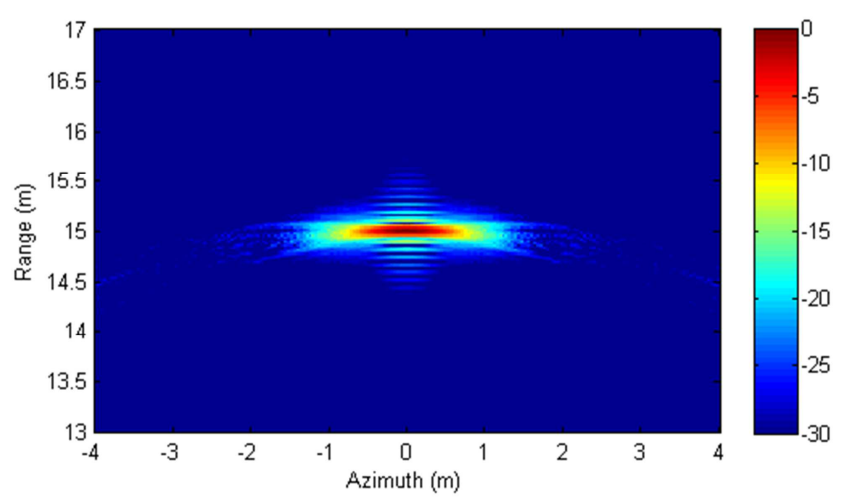

Fig. 2. PSF formed by the optimized MIMO array.

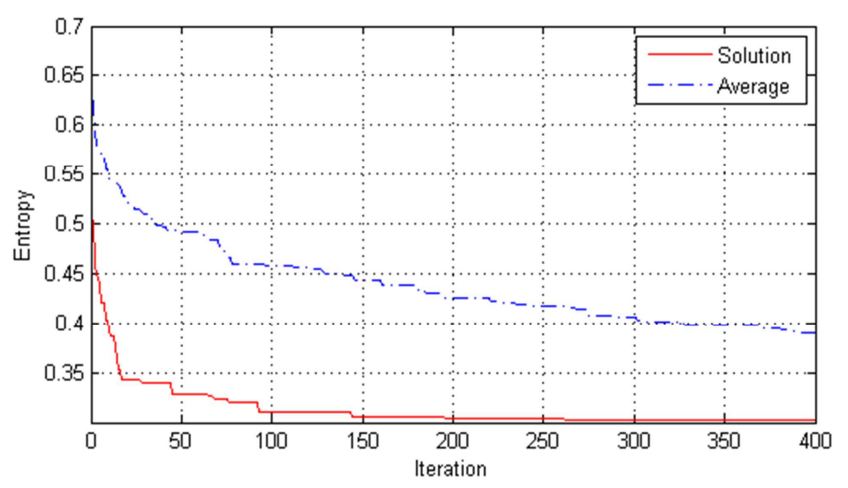

Fig. 3. Entropy changes following iteration.

\section{Conclusion}

A novel approach to design MIMO array element positions for short-range UWB imaging application is presented in this paper. The results show that the proposed approach has the potential to find better positions than random array. The proposed approach is available for short range application regardless of the space variance phenomenon. Moreover, rather than the Shannon's entropy, the approach could be also applied by changing the fitness function of the PSO due to the purpose of the imaging system. In 3D imaging cases, this approach could be used for plane MIMO array optimization.

\section{References}

[1] Edward J. Baranoski. Through-wall imaging Historical perspective and future directions [J]. Journal of the Franklin Institute. 2008, 345: 556-569.

[2] Stanley E. Borek. An Overview of Through the Wall Surveillance for Homeland Security [J]. Applied Imagery and Pattern Recognition Workshop, 2005.

[3] Laila Sakkila, Charles Tatkeu, Yassin El Hillali. UWB short range radar for road applications [J]. Physical and Chemical News, 2012, 64, p20-29.

[4] Zhuge Xiaodong. Short-range ultra-wideband imaging with multi-input multi-output arrays [D], Delft University of technology, 2010.
[5] A. Martinez-Vazquez, UWB MIMO Radar Arrays for Small Area Surveillance Applications [C], Proc. of 2nd EuCAP, 2007.

[6] L. Frazier, MDR for Law Enforcement [J], IEEE Potentials, Vol. 16, No. 5, pp. 23-26, 1998.

[7] Genyuan Wang, Imaging Through Unknown Walls Using Different Standoff Distances [J], IEEE Transactions on Signal Prossing, Vol 54, No. 10, Oct 2006, pp. 4015-4025.

[8] Francesco Soldovieri, A Multiarray Tomographic Approach for Through-Wall Imaging [J], IEEE Transactions on Geoscience and Remote Sensing, Vol, 46, No. 4, April 2008, pp. 1192-1199.

[9] Allan R. Hunt. Image Formation Through Walls Using a Distributed Radar Sensor Array [C]. Applied Imagery and Pattern Recognition Workshop, 2003.

[10] Calvin Le, Traian Dogaru, Lam Nguyen, Marc A. Ressler. Ultra-wideband (UWB) Radar Imaging of Building interior: Measurements and Predictions [C]. IEEE Transactions on Geoscience and Remote Sensing, 2009, vol. 47. 1409-1420.

[11] B. Yang, UWB MIMO Antenna Array Topology Design Using PSO for Through Dress Near-field Imaging [C], EuMA, Oct 2008, pp. 1620-1623.

[12] A. G. Yarovoy. Comparison of UWB Technologies for Human Being Detection with Radar [C], Proc. of 4th EuRAD, 2007, pp. 295-298.

[13] J. L. Schwartz, Ultrasparse, Ultrawideband Arrays, IEEE Trans. on Ultrasonics, Ferroelectrics, and Frequency Control [J], Vol. 45, No. 2, March 1998, pp. 376-393.

[14] M. Ciattaglia, Time Domain Synthesis of UWB Arrays [C], Proc. of 2nd EuCAP, 2007.

[15] Zhi Li, Tian Jin, Bo Chen, Zhimin Zhou. A coarray based MIMO array design method for UWB imaging [C]. IET International Radar Conference 2012, Glasgow, UK, 2012.

[16] Fauzia Ahmad, Saleem A. Kassam. Coarray analysis of the wide-band point spread function for active array imaging $[\mathrm{J}]$. Signal Processing, Vol. 81, pp. 99-115, 2001.

[17] Cui G, Kong L, Yang J. A Back-Projection Algorithm to Stepped-Frequency Synthetic Aperture Through-the-Wall Radar Imaging [C]. Synthetic Aperture Radar, 2007. Apsar 2007. Asian and Pacific Conference on. 2007: 123 - 126.

[18] Chen A L, Wang D W, Ma X Y. An improved BP algorithm for high-resolution MIMO imaging radar [C].Audio Language and Image Processing (ICALIP), 2010 International Conference on. 2010:1663 - 1667 .

[19] Ahmad F, Kassam S A. Coarray analysis of the wide-band point spread function for active array imaging [J]. Signal Processing, 2001, 81(1): 99-115.

[20] Dian Palupi Rini, Particle Swarm Optimization: Technique, System and Challenges [J]. International Journal of Computer Applications, vol. 14, No.1, January 2011.

[21] M. B. Ghalia, Particle Swarm Optimization with an Improved Exploration-Exploitation Balance [J], IEEE, vol. 3, 2008.

[22] J. Sok-Son,G. Thomas, Range-Doppler Radar Imaging and Motion Compensation [J], Artech House, Norwood, Mass, USA, 2001. 
[23] Daniel Flores-Tapia, An Entropy-Based Propagation Speed Estimation Method for Near-Field Subsurface Radar Imaging [J], EURASIP Journal, 2010.

[24] Pun T. A New Method for Grey-Level Picture Thresholding Using the Entropy of the Histogram [J]. Signal Processing, 1980, 2(3): 223-237.
[25] Qu L, Yang T. Investigation of Air/Ground Reflection and Antenna Beamwidth for Compressive Sensing SFCW GPR Migration Imaging [J]. IEEE Transactions on Geoscience \& Remote Sensing, 2012, 50(8): 3143-3149. 